ARTICLE

DOI: $10.1038 / s 41467-018-04843-z$

\title{
Assigning the absolute configuration of single aliphatic molecules by visual inspection
}

\author{
Daniel Ebeling $\mathbb{D}^{1}$, Marina Šekutor ${ }^{2}$, Marvin Stiefermann ${ }^{1}$, Jalmar Tschakert ${ }^{1}$, Jeremy E.P. Dahl ${ }^{3}$,
} Robert M.K. Carlson ${ }^{3}$, André Schirmeisen ${ }^{1} \&$ Peter R. Schreiner (iD ${ }^{2}$

Deciphering absolute configuration of a single molecule by direct visual inspection is the next step in compound identification, with far-reaching implications for medicinal chemistry, pharmacology, and natural product synthesis. We demonstrate the feasibility of this approach utilizing low temperature atomic force microscopy (AFM) with a CO-functionalized tip to determine the absolute configuration and orientation of a single, adsorbed [123]tetramantane molecule, the smallest chiral diamondoid. We differentiate between single enantiomers on $\mathrm{Cu}(111)$ by direct visual inspection, and furthermore identify molecular dimers and molecular clusters. The experimental results are confirmed by a computational study that allowed quantification of the corresponding intermolecular interactions. The unique toolset of absolute configuration determination combined with AFM tip manipulation opens a route for studying molecular nucleation, including chirality-driven assembly or reaction mechanisms.

\footnotetext{
${ }^{1}$ Institute of Applied Physics, Justus-Liebig University, Heinrich-Buff-Ring 16, 35392 Giessen, Germany. ${ }^{2}$ Institute of Organic Chemistry, Justus-Liebig University, Heinrich-Buff-Ring 17, 35392 Giessen, Germany. ${ }^{3}$ Stanford Institute for Materials and Energy Sciences, Stanford, CA 94305, USA. These authors contributed equally: Daniel Ebeling, Marina Šekutor. Correspondence and requests for materials should be addressed to

D.E. (email: Daniel.Ebeling@ap.physik.uni-giessen.de) or to M.Še. (email: Marina.Sekutor@org.Chemie.uni-giessen.de)

or to A.S. (email: Andre.Schirmeisen@ap.physik.uni-giessen.de) or to P.R.S. (email: prs@uni-giessen.de)
} 
ife as we know it would not exist without chirality. Homochirality of biological building blocks, e.g., L-amino acids and D-sugars, is critical for molecular recognition and has been fundamental for the evolution of life on Earth ${ }^{1}$. The mirror images (enantiomers or right-handed and left-handed forms) of the same compound can interact with living organisms in a completely different manner. As a consequence, for individual enantiomers we often observe differences in taste, smell, and in the case of pharmaceuticals, adverse medical effects ${ }^{2}$. Louis Pasteur was the first to demonstrate molecular chirality by separating right-handed and left-handed forms of tartaric acid crystals using a light microscope and then analyzing their optical activity ${ }^{3}$. At first glance, preferential crystallization seems to be a facile method for obtaining enantiomerically pure material; however, compounds capable of spontaneous symmetry breaking upon crystallization are quite rare and this phenomenon is not well understood $^{4,5}$. Formation of single-enantiomer crystals occurs so rarely because the Gibbs free energy $(\Delta G)$ of racemate crystallization is almost always negative, thereby favouring the formation of a racemic crystal containing both enantiomers ${ }^{6}$. Additionally, the chirality of crystals does not translate directly into the absolute stereochemistry of a molecule because the absolute configuration can be assigned only after fitting molecular orientation to the crystal polar axis ${ }^{7}$. Since the time of Pasteur's experiment, various analytical techniques have been perfected to help determine the absolute configurations of compounds, e.g., measurement of optical rotation, circular dichroism, X-ray analysis, NMR spectroscopic methods, etc. ${ }^{8,9}$, but to assign absolute configuration of individual molecules directly by visual inspection remains a highly attractive goal ${ }^{10}$. This would constitute a major advance for chemistry.

Previously, assignment of enantiomers without chromophores, e.g., chiral alkanes ${ }^{11,12}$ and many natural products, which display very small optical rotations, was particularly difficult and led to high uncertainties and well-documented misassignments ${ }^{13}$. Although isolation and structure elucidation of natural products has progressed dramatically ${ }^{14}$, their identification by direct observation of single molecules would eliminate some persistent problems connected with natural product isolation and/or total synthesis, e.g., limited compound availability and a necessity for further functionalization requiring additional synthetic steps. Taking into account the present need for such a direct method, we decided to use helically chiral [123] tetramantane ${ }^{15}$ as a model system for determining absolute configuration by means of visual inspection of single molecules.

[123] Tetramantane, a natural product that can be isolated from petroleum ${ }^{16}$, is a chiral alkane belonging to the class of molecules known as diamondoids (Fig. 1) ${ }^{17},{ }^{18}$. Assignment of its enantiomers is particularly challenging using the established methods described above ${ }^{15}$ because it requires multiple tedious steps including: (1) HPLC separations using a chiral stationary phase, (2) crystal growth, (3) chemical functionalization, and (4) use of a variety of analytical and theoretical techniques to unambiguously assign the structures. To underscore the challenge of assigning the absolute configuration of some molecules without classic chromophores, it is noteworthy that the $[\alpha]_{D}$ value of [123] tetramantane enantiomers is only $34^{\circ}$. Therefore, there are no interpretable absorptions in the circular dichroism (CD) spectrum and the structure could only be reliably assigned using a match of computed and measured vibrational circular dichroism spectra ${ }^{15}$. Owing to these difficulties, we decided to approach the problem differently, by using a single molecule perspective.

With methods such as scanning tunneling microscopy (STM) or atomic force microscopy (AFM) it is possible to study chirality at the molecular level ${ }^{19-21}$. In particular, the reduction of the degrees of freedom, which results from analyzing adsorbed 2D molecular layers or small 2D clusters can facilitate differentiation between enantiomers. Following this strategy, along with choosing a substrate where surface reconstruction can take place, it is sometimes possible to identify the chirality of molecular dimers even without submolecular resolution ${ }^{21}$. However, to assign the absolute configuration at the level of individual molecules submolecular resolution is essential. For example, Ernst et al. recently demonstrated the concept of stereochemical assignment for helical molecules on a metal surface using the submolecular resolution capabilities of standard $\mathrm{STM}^{10}$. Furthermore, highresolution AFM was also used to identify products of on-surface chirality transfer reactions from helicene substrates by determining the handedness of mostly planar single molecules ${ }^{22}$. In these two exemplary cases, however, the unique structure of the studied types of helical molecules facilitates their identification. Additionally, since such helicenes are non-natural aromatic hydrocarbons that typically have large optical rotations and intense CD spectra, their assignment by conventional means is straightforward. Extending the assignment of absolute configuration by direct visual inspection to single $s p^{3}$ systems would therefore represent a significant advance.

For small bulky molecules it is difficult and unreliable to assign their configuration by using standard STM measurements (i.e., without tip functionalization) and only a rough estimation of their orientation is feasible, as demonstrated for single tetramantane molecules on a $\mathrm{Au}(111)$ surface ${ }^{23,24}$. Hence, in order to study such types of molecules a technique with higher lateral resolution is needed, which can, e.g., be accomplished by functionalizing the STM/AFM tip. This so-called "bond imaging" technique has previously been introduced by Gross et al. who
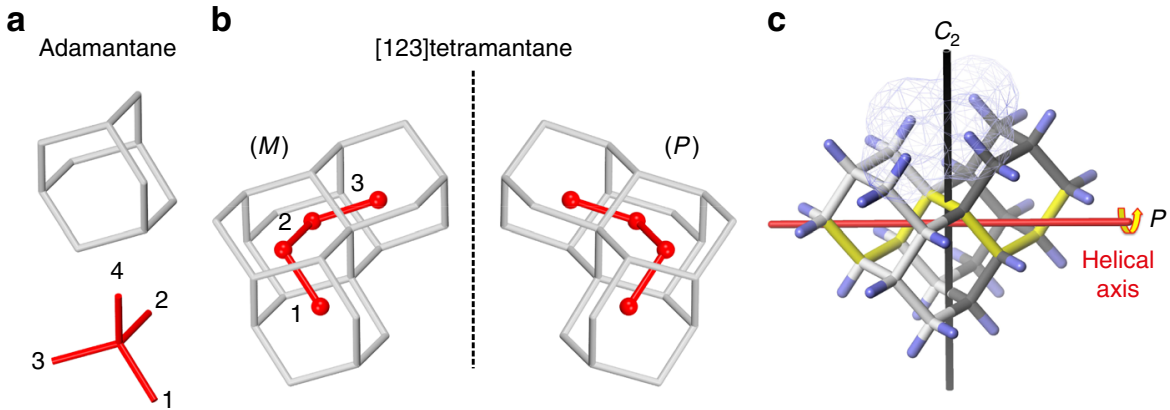

Fig. 1 Structures of adamantane and enantiomers of [123]tetramantane. a Depiction of adamantane. b (M)-enantiomer and (P)-enantiomer of [123] tetramantane with hydrogen atoms omitted for clarity (numbers 1-4 are used to indicate the directions in Cartesian space of the diamond lattice and four red spheres in molecules indicate the centers of four corresponding adamantane cages). c The $(P)$-enantiomer illustrating the helical core (bonds marked in yellow) and close contacts between the hydrogens inside the molecular groove (transparent net area) 
demonstrated that AFM resolution can be significantly enhanced by functionalizing the tip with a single CO molecule ${ }^{25}$. Similarly, proper tip functionalization can provide enhanced contrast in specific STM techniques ${ }^{26-28}$. In particular, for the study of aromatic compounds on surfaces these methods have been successfully applied to identify chemical structures, length, and order of intramolecular and intermolecular bonds, molecular assembly mechanisms, on-surface reaction pathways, and even chiralityrelated phenomena ${ }^{22,25,29-38}$

Here we applied this technique to differentiate between single enantiomers of [123]tetramantane on a $\mathrm{Cu}(111)$ surface and determine their absolute configuration. This is the first time single enantiomers of naturally occurring bulky molecules have been distinguished solely by direct visual inspection. In addition, we studied the self-assembly of chiral molecules on metal surfaces and imaged [123]tetramantane dimers and small clusters which may act as centers for crystal nucleation.

\section{Results and Discussion}

STM/AFM imaging. In order to investigate single [123]tetramantanes, the racemic mixture was sublimed onto a cold $\mathrm{Cu}(111)$ surface (below $\approx 15 \mathrm{~K}$ ) through the opened temperature shields of the low-temperature STM/AFM instrument. At higher temperatures tetramantane tends to form islands ${ }^{23}, 36$, which are caused by a low diffusion barrier paired with strong intermolecular London dispersion (LD) interactions ${ }^{36}$. Figure 2 shows STM (a) and AFM (b-d) scans of single [123]tetramantanes that were measured at $5 \mathrm{~K}$ with a $\mathrm{CO}$ functionalized AFM tip ${ }^{25}$. The appearance of the molecules in two different imaging modes is rather different. In the STM mode the molecules appear as cloudlike structures that exhibit sub-molecular features (Fig. 2a). Since the STM contrast depends strongly on the local density of states, these features in general do not allow for direct identification of molecular structures. The AFM contrast, however, is mainly determined by short-range repulsive interactions, i.e., Pauli exchange repulsion and electrostatic interactions ${ }^{25}, 39$, which are known to produce a more detailed view of the chemical structure of adsorbed molecular species.

For 3D objects such as bulky cage molecules, however, direct identification of their structures and orientations on the surface is
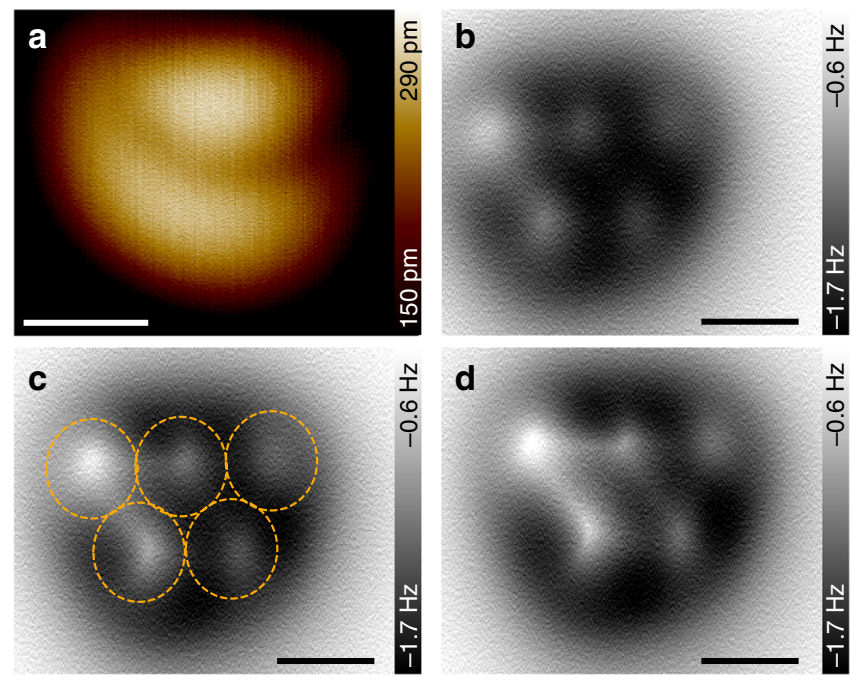

Fig. 2 Single [123]tetramantane molecules on a Cu(111) surface. a Images obtained in STM scan mode. b-d Images obtained by constant height AFM scans. Parameters: a $U=200 \mathrm{mV}, I=10 \mathrm{pA}, \mathbf{b}-\mathbf{d} U=-0.5 \mathrm{mV}, \Delta z=$ $170 \mathrm{pm}(\mathbf{b}), 160 \mathrm{pm}$ (c), $150 \mathrm{pm}$ (d), respectively. Scale bars: a $0.5 \mathrm{~nm}$, b-c $0.3 \mathrm{~nm}$ not straightforward ${ }^{30,38,40-43}$, although some recent advances in characterizing aliphatic moieties have been made ${ }^{36}, 43$. The CO molecule at the tip, which facilitates the submolecular contrast, is flexible and provokes distortions in the image contrast ${ }^{25}, 30,41$. This flexibility of the CO tip leads to a force-dependent tilting that can impede the identification of $3 \mathrm{D}$ objects during constant height scanning. Therefore, the formation of the image contrast has to be addressed thoroughly at different imaging heights in order to resolve the atomic structure and determine the precise orientation of the molecules. Recently, we were able to image [121] tetramantanes with atomic resolution and determine their precise orientation on the surface ${ }^{36}$, so this method can be applied in a similar way to the [123]tetramantanes studied here.

In Fig. 2b-d three AFM images of the same molecule are depicted, which were determined at three different heights above the $\mathrm{Cu}$ substrate. All images were obtained in the constant height mode and the $\Delta z$-offset is given with regard to the tip height over the $\mathrm{Cu}$ surface in tunneling feedback $(U=200 \mathrm{mV}, I=10 \mathrm{pA})$. Displayed is the frequency shift of the oscillating tuning fork sensor vs. its $x / y$-position. While repulsive tip-sample interactions lead to positive frequency shifts (bright colors), attractive interactions lead to negative frequency shifts (dark colors). The observed image contrast changes significantly when approaching the CO-tip to the sample surface. Initially, at distances relatively far away from the molecule, a dark region appears that is due to attractive LD interactions (not shown). Closer to the surface (Fig. 2b, c), the images reveal five bright spots, whose arrangement bears similarity to the Olympic rings (as indicated by dashed orange circles in $\mathrm{c}$ ). When further approaching the surface artificial lines appear between the bright spots that are caused by the previously mentioned flexibility of the $\mathrm{CO}$ molecule (Fig. 2d). For closer tip-sample distances these effects get more severe and make the images non-analyzable.

Determining the absolute configuration. In the following we demonstrate that the observed Olympic ring pattern of five bright spots allows us to determine the precise orientation of the individual [123]tetramantane molecules and their absolute configuration. Fig. 3 shows three different orientations of [123] tetramantane that have been observed on the $\mathrm{Cu}(111)$ surface (left ( $a, d, g)$, middle (b, e, h), and right column (c, f, i)). The first two rows depict side and top views of the corresponding computed orientations (for details about the used GFN-xTB 44 computational method vide infra). To each of the side views a semi-transparent plane was added, indicating the imaging planes of the corresponding AFM experiment. AFM images for each of the observed molecular orientations are depicted in the third row. Hydrogen atoms facing the $\mathrm{Cu}(111)$ surface are marked in blue, while hydrogen atoms within the imaging plane are marked in orange. All other hydrogen atoms are shown as white sticks for clarity. Each orientation leads to a characteristic pattern of hydrogen atoms within the imaging plane, which resemble the shape of the Olympic rings $(\mathrm{d}, \mathrm{g})$, a triangle $(\mathrm{e}, \mathrm{h})$, and a rhombus (f, i).

The majority of imaged molecules is found in the Olympic ring pattern ( $\mathrm{a}, \mathrm{d}, \mathrm{g})$ and the other two orientations are rarely observed. These orientations may be found after accidentally manipulating the molecules with the tip since this can happen during STM measurement with high tunneling setpoint or if the tip approaches the surface too closely during AFM constant height scanning. This observation is in agreement with our computational results, since the computed Gibbs energies at $15 \mathrm{~K}$ show that the orientation in the left column (Olympic ring pattern) is by 11.1 and $10.2 \mathrm{kcal} \mathrm{mol}^{-1}$ more favourable than the orientations in the middle and right columns, respectively (see 
a

Hydrogen in imaging plane

Hydrogen in surface plane
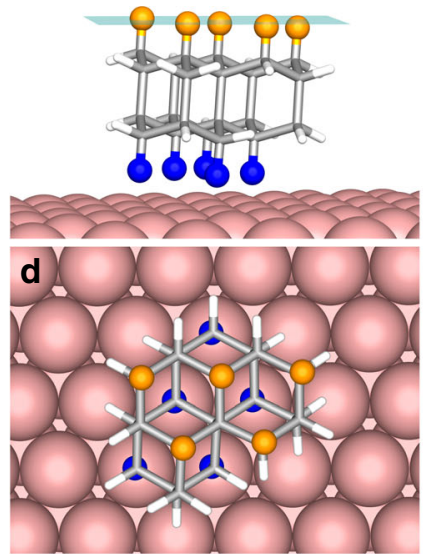

b

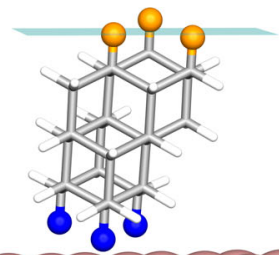

C
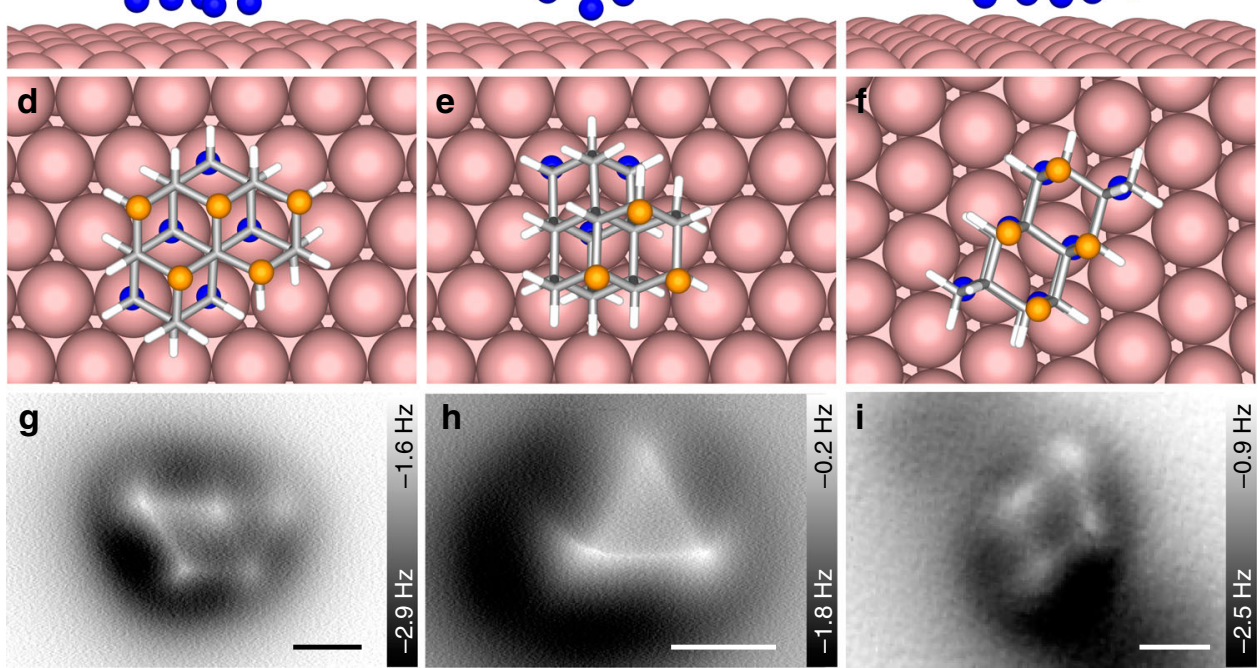

Fig. 3 Three different orientations of (M)-[123]tetramantane. In the first two rows side (a-c) and top views (d-f) of the corresponding computed orientations are depicted. The planes in the first row indicate the imaging plane of the corresponding AFM scans (shown in the third row, $\mathbf{g}-\mathbf{i}$ ). Hydrogen atoms in the imaging and surface planes are marked in orange and blue, respectively. Each orientation results in a characteristic pattern of hydrogen atoms within the imaging plane, which resemble the shape of the Olympic rings $(\mathbf{d}, \mathbf{g})$, a triangle $(\mathbf{e}, \mathbf{h})$, and a rhombus (f, i). Scale bars: $0.3 \mathrm{~nm}$

Supplementary Table 7). In general, the adsorption of $(M)$-[123] tetramantane on a $\mathrm{Cu}(111)$ surface was found to be a favourable process, e.g., at $15 \mathrm{~K}$ the orientation in the left column is stabilized by $-30.3 \mathrm{kcal} \mathrm{mol}^{-1}$ upon interaction of the isolated tetramantane molecule with the modelled copper slab.

In the following we will focus on identifying the absolute configuration of two [123] tetramantane enantiomers by visual inspection. Since the majority of the molecules are found with the Olympic ring pattern facing upwards (left column), we will describe the identification process using this orientation as an example.

Fig. 4a-c shows side view and two top view sketches of the $(M)$ enantiomer and the $(P)$-enantiomer. In $\mathrm{b}$ and $\mathrm{c}$ two specific hydrogen atoms that are located $\approx 130 \mathrm{pm}$ below the imaging plane are marked in red color, respectively. While these two specific hydrogens are located at the right side above the Olympic ring pattern for the $(M)$-type molecule, they are located at the left side for the $(P)$-type molecule (see top views b and c). Hence, for an unambiguous identification of the absolute configurations the positions of two specific hydrogen atoms, which reside $\approx 130 \mathrm{pm}$ below the imaging plane, have to be determined. Figure $4 \mathrm{~d}$ and e shows two constant height AFM scans of two different [123] tetramantanes. These scans reveal that the molecules are slightly tilted with respect to the surface plane, i.e., one side of the Olympic ring pattern appears slightly brighter than the other, as indicated by the white and dark arrows. To identify the absolute configuration of the molecules by imaging the specific hydrogens, we varied the height of the imaging plane during scanning as indicated in the sketch in Fig. 4a. The resulting AFM images are presented in Fig. $4 \mathrm{f}$ and g. Both scans were started at the bottom of the image and scanned in upward direction. After the tip has passed the Olympic ring pattern, the $\Delta z$-offset was gradually reduced by $\approx 130 \mathrm{pm}$. The two red dashed lines indicate the image region where the offset was reduced. Apparently, both images reveal characteristic features (see red dashed oval in $\mathrm{f}$ ) at the positions where the two specific hydrogen atoms are expected, i.e., at the right and left sides above the Olympic ring patterns. Herewith, we can unambiguously identify the $(M)$-enantiomer in Fig. $4 \mathrm{f}$ and the $(P)$-enantiomer in Fig. $4 \mathrm{~g}$ (see also molecular overlays in Fig. $4 \mathrm{~h}$ and i).

After performing this identification procedure, it is possible to distinguish between the two enantiomers by standard constant height AFM scans (i.e., without custom height profile). Since the molecules are not perfectly parallel to the surface plane, the brighter side of the Olympic ring pattern indicates the absolute configuration (see black and white arrows in Fig. $4 \mathrm{~d}-\mathrm{g}$ ). Presumably the observed tilting of the molecules is caused by their $C_{2}$-symmetry. The five hydrogen atoms within the Olympic ring pattern in the surface plane (see blue hydrogens in Fig. $4 \mathrm{a}-\mathrm{c}$ ) are rotated by $60^{\circ}$ with respect to the Olympic ring pattern in the imaging plane (orange hydrogens). Two specific hydrogens (red atoms), which are indicators of handedness, also have corresponding counterparts close to the surface (red dashed circles in Fig. $4 \mathrm{a}-\mathrm{c})$. For the $(M)$-enantiomers and $(P)$-enantiomers, these hydrogens are located at opposite sides (cf. Fig. $4 \mathrm{~b}$ and $c$ ). Interactions between these hydrogens and the $\mathrm{Cu}(111)$ surface are believed to pull down the molecules at the corresponding edges, provoking the observed tilting (see Supplementary Fig. 1 for more details).

Computation of on-surface structures. To further confirm such tilting of [123] tetramantane on $\mathrm{Cu}(111)$ and to provide an estimate of the tilting angle, we performed semiempirical computations using the GFN-xTB approach ${ }^{44}$. This modern method allows for computation of large systems with the advantage of also taking non-covalent interactions into account. Since LD 


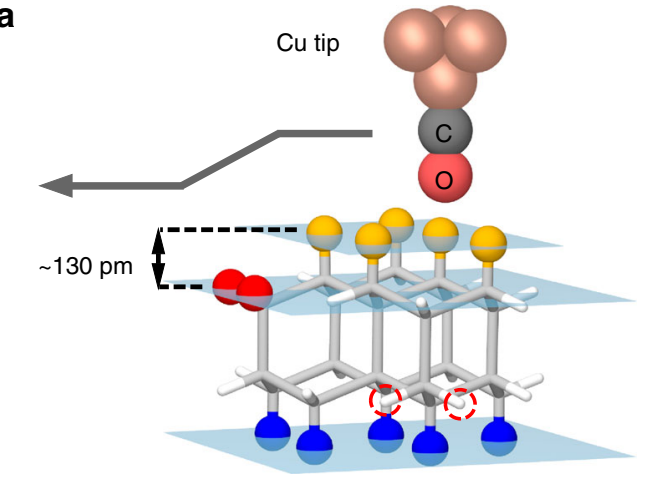

Hydrogen in imaging plane

Hydrogen in surface plane

Specific hydrogen $\sim 130 \mathrm{pm}$ below imaging plane

b

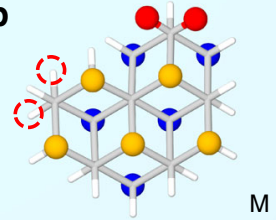

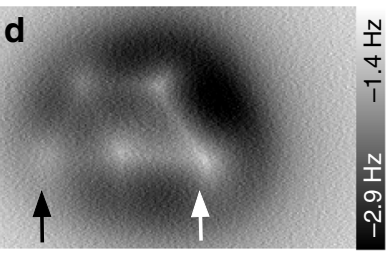
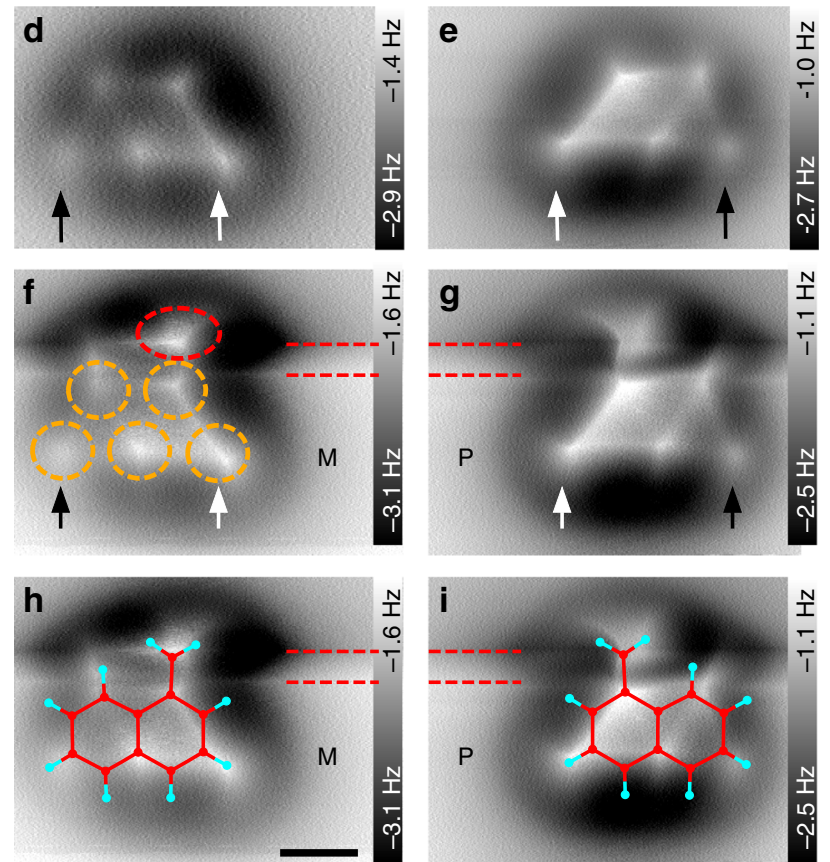

Fig. 4 Adsorption structure of [123]tetramantanes. a Side view sketch of (M)-[123]tetramantane with the Olympic ring pattern of hydrogen atoms facing upwards (see five orange hydrogens). The absolute configuration can be determined by locating two specific hydrogen atoms (red atoms) $\approx 130$ pm below the imaging plane. b, c Top view sketches of $(M)$-[123]tetramantane and ( $P$ )-[123]tetramantane showing the arrangement of Olympic ring patterns in the imaging and surface planes (orange and blue atoms) and the specific hydrogen atoms (red atoms and red dashed circles). $\mathbf{d}$, e Constant height AFM scans of two different [123]tetramantanes. White and black arrows indicate the brighter (higher) and darker (lower) sides of the molecules, respectively. $\mathbf{f}$, $\mathbf{g}$ Same molecules imaged with custom height profile, i.e., in the region between the two dashed red lines the imaging height was linearly decreased by $\approx 130$ $\mathrm{pm}$. Both scans were started in the bottom of the images. Both images reveal a bright halo (red dashed oval in $\mathbf{f}$ ), which is either located on the right or the left side of the Olympic ring pattern (dashed orange circles). h, i Corresponding AFM scans with overlaid molecular structures of (M)-[123]tetramantane and $(P)$-[123]tetramantane. For clarity, only the plane containing the two specific hydrogens is plotted. Please note that the specific hydrogens are either connected to the $\mathrm{C}-12$ or C-22 sites (according to IUPAC designated carbon numbers for [123]tetramantane). Scale bar: 0.3 nm

interactions play a vital role in the behavior of tetramantane molecules ${ }^{36}$, GFN-xTB that includes the well-established D3 dispersion correction was a method of choice for our system. As a sufficient representation of the $\mathrm{Cu}(111)$ surface we took a copper slab consisting of $216 \mathrm{Cu}$ atoms, with dimensions of $18 \times 18 \times 5$ $\AA$. After introducing the corresponding orientation of tetramantane on the $\mathrm{Cu}$ slab and freezing the copper lattice, geometry optimizations and frequency analyses were performed. The obtained geometries along with their coordinates and corresponding energies are given in the Supplementary Information and the Supplementary Data (Supplementary Fig. 1, Supplementary Table 7 , and Supplementary Data Set 3$)$. Herewith, we successfully confirm that $(M)$ and $(P)$-type molecules are tilted in opposite directions and we found tilting angles on the order of $4-5^{\circ}$ with regard to the $x / y$ plane.

Note that the shown molecular orientations correspond to local minima on the potential energy surface, i.e., other orientations with similar adsorption energies coexist. However, from our AFM experiments we can clearly infer the orientation of the Olympic ring pattern with regard to the $\mathrm{Cu}(111)$ lattice (see Supplementary Fig. 2). Our AFM images reveal that the Olympic ring pattern aligns to the crystallographic [1-10] direction (and equivalent [-101] and [01-1] directions), hence [123]tetramantanes are snapping to the $\mathrm{Cu}(111)$ lattice. We also used the tip as a manipulation tool in order to rotate the molecules. The manipulations result in rotations that correspond to integer multiples of $60^{\circ}$. Furthermore, the tilting with regard to the $x / y$ plane was preserved after rotational manipulation, which supports the rationale that the observed tilting is not caused by a sometimes observed slight asymmetry of the CO-tip. Hence, from a number of computed orientations that correspond to different local minima we chose those that are most comparable to our experimental results.

Imaging of dimers and small clusters. Next we applied the bond imaging method to different dimers and a small cluster of [123] tetramantanes. Fig. 5 depicts $(P, P)(\mathrm{a}, \mathrm{b}),(M, P)(\mathrm{c}, \mathrm{d})$, and $(M, M)$ $(\mathrm{e}, \mathrm{f})$ dimers. The absolute configuration of each molecule was identified by the observed molecular tilting with regard to the $x / y$ plane (see black and white arrows). Overlays with the corresponding molecular structures are depicted in the right column of Fig. 5b, d, f. In the case of molecular dimers the Olympic ring patterns of the individual molecules also align with the crystallographic [1-10] direction (see Supplementary Fig. 3). Precise knowledge about molecular orientation allows for determination of close contacts between two molecules within the dimers. Such information can also be used to quantify LD interactions between the molecules and pinpoint the onset of the crystallization process $^{36}$. Using these structural data as a starting point, we performed a detailed computational study for two series of dispersion-bound complexes consisting of two enantiomers, $(M$, $P)$ and $(M, M)$-[123]tetramantanes, respectively (details are provided in Supplementary Figs. 4, 5 Supplementary Tables 1-6). The obtained results confirmed that dimer formation is indeed an energetically favourable process driven by LD and the strength of these interactions was successfully quantified.

A cluster of six [123]tetramantanes is depicted in Fig. $6 a$ and b. As in the previous figures, the AFM scans allow the assignment of 

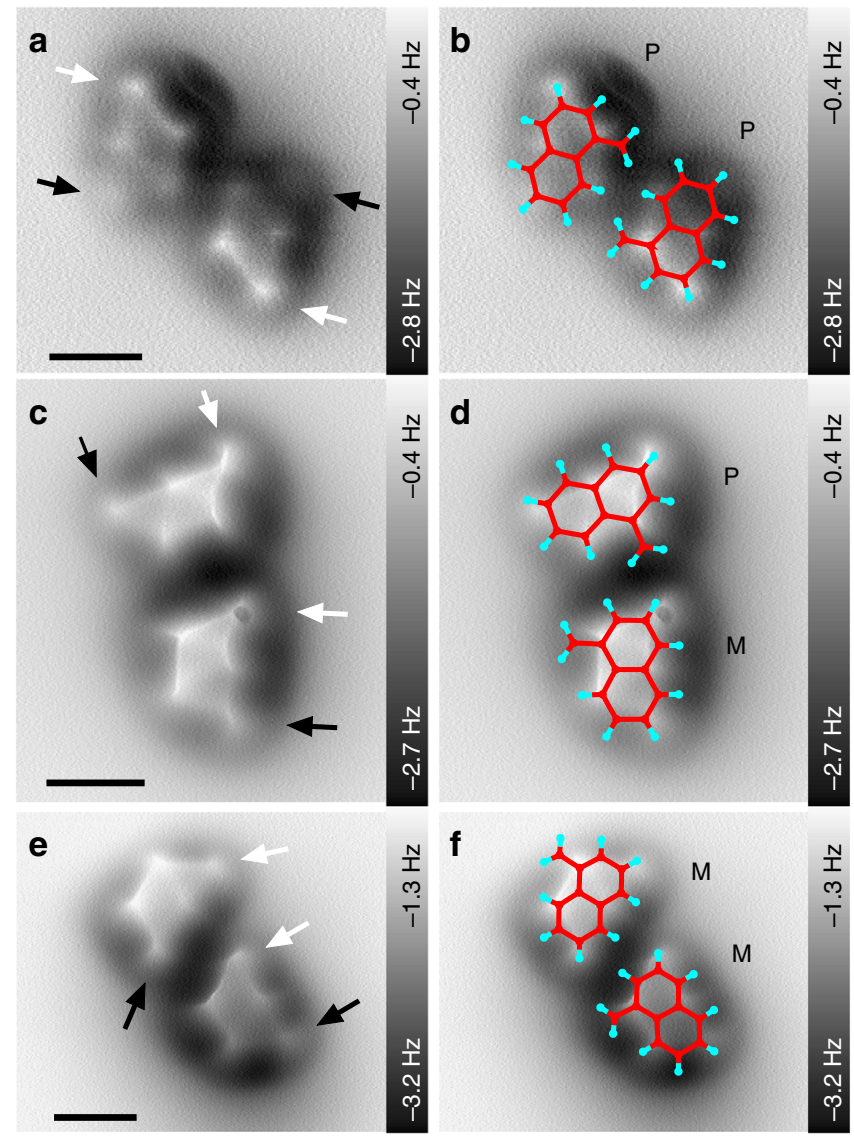

Fig. 5 Constant height AFM scans of [123]tetramantane dimers. a, b $(P, P)$ [123]tetramantane dimers. $\mathbf{c}, \mathbf{d}(M, P)$-[123]tetramantane dimers. e, f $(M$, M)-[123]tetramantane dimers. Tilting of the molecules is indicated by black and white arrows (as in Fig. 4). b, d, f AFM scans overlaid with molecular structures. For clarity only the plane containing the two specific hydrogens is plotted (cf. Fig. 4). Scale bars: $0.5 \mathrm{~nm}$

absolute configuration and the precise determination of molecular orientation. An overlay of the molecular structures is shown in $(c, d)$. Images (a, c) were taken before, while images (b, d) were obtained after deliberately manipulating the molecules with the AFM tip. Therefore, the molecular cluster has been imaged in STM mode while the gap voltage and tunneling current were systematically changed until manipulation of the molecules was observed. Fig. $6 \mathrm{~b}$ reveals that five of the six molecules have been rotated by this procedure. Comparing the arrangement of the molecules before and after manipulation reveals very similar patterns of close contacts between the molecules, showing that intermolecular $\mathrm{LD}$ interactions have a significant influence on the process of molecular assembly.

Finally, we discuss how cluster formation depends on the interplay between intermolecular LD interactions and molecule-surface interactions. The observed snapping of single [123]tetramantanes and molecular dimers to the $\mathrm{Cu}$ lattice demonstrates that molecule-surface interactions play a significant role in on-surface assembly (see Supplementary Figs. 2, 3). The imaged molecular cluster, however, reveals a slight deviation regarding the molecular orientations, i.e., in Fig. 6a the three observed orientations of the Olympic ring patterns (see red lines indicated with numbers 1-3) are not perfectly parallel to the crystallographic [1-10], [10-1], and [01-1] directions (see three red arrows). This indicates that intermolecular LD interactions start to have an increased role in molecular assembly as more bulky molecules are being added to the cluster.

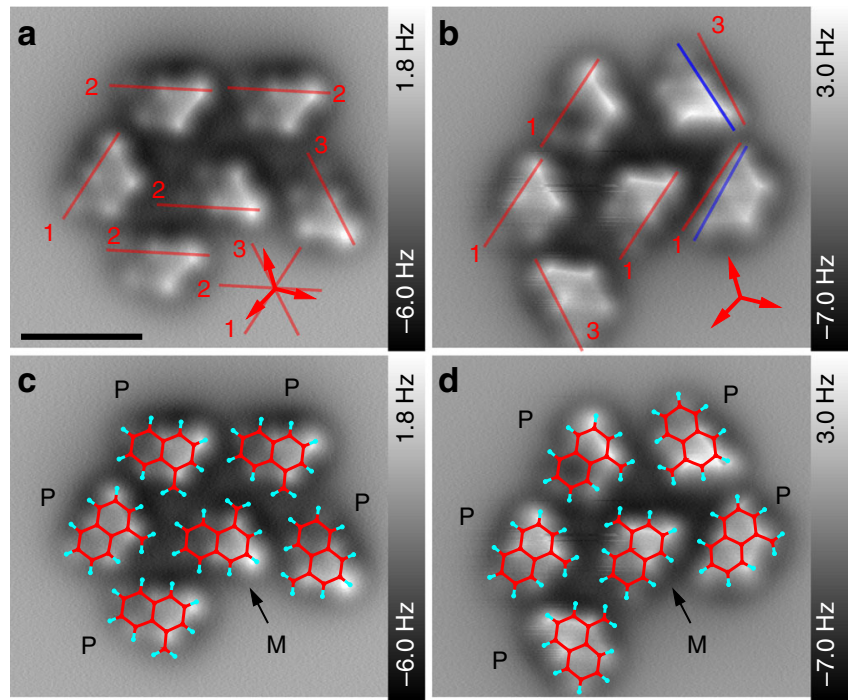

Fig. 6 Constant height AFM scans of a molecular cluster consisting of [123] tetramantane. a Scan before manipulation by AFM tip. b Scan after manipulation by AFM tip. The numbered red lines indicate the three main directions of molecular alignment. The three red arrows indicate the crystallographic [0-11], [10-1], and [-110] directions. The two blue lines in b indicate molecules that were not rotated by inter multiples of $60^{\circ}$.

c, d AFM scans overlaid with molecular structures. The cluster contains five $(P)$-enantiomers and one $(M)$-enantiomer. For clarity only the plane containing the two specific hydrogens is plotted (see Fig. 4). Scale bar: $1 \mathrm{~nm}$

This observation is even more pronounced after deliberately manipulating the molecules with the $\mathrm{CO}$ tip. Fig. $6 \mathrm{~b}$ shows that three out of five manipulated molecules are rotated by integer multiples of approx. $60^{\circ}$, while two molecules are found to be misaligned by a few degrees (see blue lines). The two blue lines show an even stronger deviation from the crystallographic [1-10] directions than the three red lines. Hence, for the studied molecular cluster intermolecular LD interactions start to dominate the assembling process, while molecule-surface interactions play here only a minor role. This is in line with our previous results, which show that $\mathrm{LD}$ interactions can direct the self-assembly of [121]tetramantane within larger clusters (approx. 10 molecules) and big islands ${ }^{36}$. Furthermore, the possibility to directly assign the precise orientation and the absolute configuration of the molecules paired with the manipulation capabilities of scanning probe techniques will allow future studies of chirality driven assembly mechanisms at a new level of accuracy.

To summarize, we assigned by direct visual inspection the absolute configuration and orientation of adsorbed [123]tetramantanes on $\mathrm{Cu}(111)$ using low temperature AFM with a $\mathrm{CO}$ functionalized tip. The approach was successfully applied to single molecules, molecular dimers, and small molecular clusters. We determined the intermolecular arrangement of enantiomers with atomic precision and supported the experimental findings with a systematic computational study. The present approach for assigning absolute configurations of chiral molecules can thus be considered as an emerging tool for studying molecular recognition and reactivity of chiral compounds on surfaces, capable of providing a new level of sophistication.

\section{Methods}

STM/AFM measurements. [123] Tetramantane was isolated from petroleum and purified by multiple HPLC separations as described previously ${ }^{16}$. We used an ultrahigh vacuum low temperature STM/AFM (ScientaOmicron, Germany) with a base 
pressure below $1 \times 10^{-10} \mathrm{mbar}$. The $\mathrm{Cu}(111)$ single crystal substrate (MaTecK, Germany) was cleaned prior to the experiment, by multiple (up to 100) cycles of $\mathrm{Ar}^{\bullet+}$ sputtering $\left(1.5 \mathrm{keV}, 3-6 \times 10^{-6} \mathrm{mbar}, 3-4 \mu \mathrm{A}\right)$ and subsequent annealing (up to $1000 \mathrm{~K}$ ). For evaporation a small amount of molecule powder was inserted into a stainless steel tube that was attached to an Omicron sample plate and kept at room temperature ${ }^{45}$. The molecules were deposited onto the $\mathrm{Cu}$ surface (below $15 \mathrm{~K}$ ) through the opened temperature shields of the STM/AFM instrument. All measurements were performed with the tip connected to ground and the sample connected to bias voltage at a temperature of $5 \mathrm{~K}$. Commercial (ScientaOmicron) and homemade ${ }^{46}$ sensors with tungsten tips have been used (resonant frequency = 19.3 or $27.0 \mathrm{kHz}, Q$-factor $=15,000-30,000$, oscillation amplitude $=60-160 \mathrm{pm}$ ), $\mathrm{CO}$ molecules have been picked up from the $\mathrm{Cu}$ surface using the recipe of Bartels et al. ${ }^{47}$ or by applying voltage pulses of $3-4 \mathrm{~V}$.

Computations. Semiempirical computations of [123]tetramantane molecules on a $\mathrm{Cu}(111)$ surface were performed using the GFN-xTB approach developed by Grimme et $\mathrm{al}^{44}$. A copper crystal surface cut-out with dimensions of $18 \times 18 \times 5 \AA$ consisting of $216 \mathrm{Cu}$ atoms was taken and the atoms were frozen to simulate the copper lattice. Tetramantane molecules of the corresponding orientation observed in the AFM images (Olympic rings, triangle, and rhombus) were placed on the copper slab, optimized and their frequency analyses were performed. The obtained geometries were then compared with the structural parameters obtained from AFM imaging.

Data availability. The data supporting the findings of this study are available within the paper and its Supplementary Information files.

Received: 4 March 2018 Accepted: 30 May 2018

Published online: 20 June 2018

\section{References}

1. Blackmond, D. G. The origin of biological homochirality. Cold Spring Harb. Perspect. Biol. 2, a002147 (2010).

2. Kim, J. H. \& Scialli, A. R. Thalidomide: the tragedy of birth defects and the effective treatment of disease. Toxicol. Sci. 122, 1-6 (2011).

3. Flack, H. Louis Pasteur's discovery of molecular chirality and spontaneous resolution in 1848 , together with a complete review of his crystallographic and chemical work. Acta Crystallogr. A Found. Adv. 65, 371-389 (2009).

4. Levilain, G. \& Coquerel, G. Pitfalls and rewards of preferential crystallization. CrystEngComm 12, 1983-1992 (2010).

5. Saito, Y. \& Hyuga, H. Colloquium: homochirality: symmetry breaking in systems driven far from equilibrium. Rev. Mod. Phys. 85, 603-621 (2013).

6. Toxvaerd, S. Homochirality in bio-organic systems and glyceraldehyde in the formose reaction. J. Biol. Phys. 31, 599-606 (2005).

7. Weissbuch, I. et al. Centrosymmetric crystals for the direct assignment of the absolute configuration of chiral molecules. Application to the $\alpha$-amino acids by their effect on glycine crystals. J. Am. Chem. Soc. 105, 6615-6621 (1983).

8. Seco, J. M., Quiñoá, E. \& Riguera, R. The assignment of absolute configuration by NMR. Chem. Rev. 104, 17-118 (2004).

9. Allenmark, S. \& Gawronski, J. Determination of absolute configuration-an overview related to this Special Issue. Chirality 20, 606-608 (2008).

10. Ernst, K.-H. et al. Pasteur's experiment performed at the nanoscale: manual separation of chiral molecules, one by one. Nano Lett. 15, 5388-5392 (2015).

11. Masarwa, A. et al. Synthesis and stereochemical assignment of crypto-optically active ${ }^{2} \mathrm{H}_{6}$-neopentane. Angew. Chem. Int. Ed. 54, 13106-13109 (2015).

12. McCann, D. M., Stephens, P. J. \& Cheeseman, J. R. Determination of absolute configuration using density functional theory calculation of optical rotation: chiral alkanes. J. Org. Chem. 69, 8709-8717 (2004).

13. Nicolaou, K. C. \& Snyder, S. A. Chasing molecules that were never there: misassigned natural products and the role of chemical synthesis in modern structure elucidation. Angew. Chem. Int. Ed. 44, 1012-1044 (2005).

14. Bucar, F., Wube, A. \& Schmid, M. Natural product isolation-how to get from biological material to pure compounds. Nat. Prod. Rep. 30, 525-545 (2013).

15. Schreiner, P. R. et al. [123]Tetramantane: parent of a new family of $\sigma$ helicenes. J. Am. Chem. Soc. 131, 11292-11293 (2009).

16. Dahl, J. E., Liu, S. G. \& Carlson, R. M. K. Isolation and structure of higher diamondoids, nanometer-sized diamond molecules. Science 299, 96-99 (2003).

17. Schwertfeger, H., Fokin, A. A. \& Schreiner, P. R. Diamonds are a chemist's best friend: diamondoid chemistry beyond adamantane. Angew. Chem. Int. Ed. 47, 1022-1036 (2008).
18. Gunawan, M. A. et al. Diamondoids: functionalization and subsequent applications of perfectly defined molecular cage hydrocarbons. New J. Chem. 38, 28-41 (2014).

19. Lopinski, G. P., Moffatt, D. J., Wayner, D. D. M. \& Wolkow, R. A. Determination of the absolute chirality of individual adsorbed molecules using the scanning tunnelling microscope. Nature 392, 909-911 (1998).

20. Böhringer, M., Morgenstern, K., Schneider, W.-D. \& Berndt, R. Separation of a racemic mixture of two-dimensional molecular clusters by scanning tunneling microscopy. Angew. Chem. Int. Ed. 38, 821-823 (1999).

21. Kuhnle, A., Linderoth, T. R., Hammer, B. \& Besenbacher, F. Chiral recognition in dimerization of adsorbed cysteine observed by scanning tunnelling microscopy. Nature 415, 891-893 (2002).

22. Stetsovych, O. et al. From helical to planar chirality by on-surface chemistry Nat. Chem. 9, 213-218 (2016).

23. Wang, Y. et al. Spatially resolved electronic and vibronic properties of single diamondoid molecules. Nat. Mater. 7, 38-42 (2008)

24. Pechenezhskiy, I. V. et al. Infrared spectroscopy of molecular submonolayers on surfaces by infrared scanning tunneling microscopy: tetramantane on $\mathrm{Au}$ (111). Phys. Rev. Lett. 111, 126101 (2013).

25. Gross, L., Mohn, F., Moll, N., Liljeroth, P. \& Meyer, G. The chemical structure of a molecule resolved by atomic force microscopy. Science 325, 1110-1114 (2009).

26. Weiss, C. et al. Imaging Pauli repulsion in scanning tunneling microscopy. Phys. Rev. Lett. 105, 086103 (2010).

27. Chiang, C.-1, Xu, C., Han, Z. \& Ho, W. Real-space imaging of molecular structure and chemical bonding by single-molecule inelastic tunneling probe. Science 344, 885-888 (2014).

28. de la Torre, B. et al. Submolecular resolution by variation of the inelastic electron tunneling spectroscopy amplitude and its relation to the AFM/STM signal. Phys. Rev. Lett. 119, 166001 (2017).

29. Gross, L. et al. Organic structure determination using atomic-resolution scanning probe microscopy. Nat. Chem. 2, 821-825 (2010).

30. Gross, L. et al. Bond-order discrimination by atomic force microscopy. Science 337, 1326-1329 (2012).

31. de Oteyza, D. G. et al. Direct imaging of covalent bond structure in singlemolecule chemical reactions. Science 340, 1434-1437 (2013).

32. Zhang, J. et al. Real-space identification of intermolecular bonding with atomic force microscopy. Science 342, 611-614 (2013).

33. Kawai, S. et al. Extended halogen bonding between fully fluorinated aromatic molecules. ACS Nano 9, 2574-2583 (2015).

34. Pavliček, N. et al. On-surface generation and imaging of arynes by atomic force microscopy. Nat. Chem. 7, 623-628 (2015).

35. Riss, A. et al. Imaging single-molecule reaction intermediates stabilized by surface dissipation and entropy. Nat. Chem. 8, 678-683 (2016).

36. Ebeling, D. et al. London dispersion directs on-surface self-assembly of [121] tetramantane molecules. ACS Nano 11, 9459-9466 (2017).

37. Han, Z. et al. Imaging the halogen bond in self-assembled halogenbenzenes on silver. Science 358, 206-210 (2017).

38. Zint, S. et al. Imaging successive intermediate states of the on-surface Ullmann reaction on $\mathrm{Cu}(111)$ : role of the metal coordination. ACS Nano 11, 4183-4190 (2017).

39. Hapala, P., Temirov, R., Tautz, F. S. \& Jelínek, P. Origin of high-resolution IETS-STM images of organic molecules with functionalized tips. Phys. Rev. Lett. 113, 226101 (2014).

40. Pavliček, N. et al. Atomic force microscopy reveals bistable configurations of dibenzo[a,h]thianthrene and their interconversion pathway. Phys. Rev. Lett. 108, 086101 (2012)

41. Hapala, P. et al. Mechanism of high-resolution STM/AFM imaging with functionalized tips. Phys. Rev. B 90, 085421 (2014).

42. Ebeling, D. et al. Chemical bond imaging using higher eigenmodes of tuning fork sensors in atomic force microscopy. Appl. Phys. Lett. 110, 183102 (2017).

43. Schuler, $\mathrm{M}$ et al. Characterizing aliphatic moieties in hydrocarbons with atomic force microscopy. Chem. Sci. 8, 2315-2320 (2017).

44. Grimme, S., Bannwarth, C. \& Shushkov, P. A Robust and accurate tight binding quantum chemical method for structures, vibrational frequencies, and noncovalent interactions of large molecular systems parametrized for all spdblock elements ( $\mathrm{Z}=1-86)$. J. Chem. Theory Comput. 13, 1989-2009 (2017).

45. Zint, S., Ebeling, D., Ahles, S., Wegner, H. A. \& Schirmeisen, A. Subsurfacecontrolled angular rotation: triphenylene molecules on $\mathrm{Au}(111)$ substrates. J. Phys. Chem. C 120, 1615-1622 (2016).

46. Falter, J., Langewisch, G., Hölscher, H., Fuchs, H. \& Schirmeisen, A. Field ion microscopy characterized tips in noncontact atomic force microscopy: quantification of long-range force interactions. Phys. Rev. B 87, 115412 (2013).

47. Bartels, L., Meyer, G. \& Rieder, K. H. Controlled vertical manipulation of single $\mathrm{CO}$ molecules with the scanning tunneling microscope: a route to chemical contrast. Appl. Phys. Lett. 71, 213-215 (1997). 


\section{Acknowledgements}

We acknowledge financial support from the Deutsche Forschungsgemeinschaft (DFG) via the GRK (Research Training Group) 2204 "Substitute Materials for Sustainable Energy Technologies". This project was also supported by the Laboratory of Materials Research (LaMa) of JLU and the LOEWE program of excellence of the Federal State of Hessen (project initiative STORE-E). The work was also supported in part (for J.E.P.D., R.M.K.C., and P.R.S.) by the US Department of Energy, Office of Science, Basic Energy Sciences, Materials Sciences and Engineering Division, under contract DE-AC0276SF00515. M.Š. thanks the Alexander-von-Humboldt Foundation for a Humboldt Research Fellowship for Postdoctoral Researchers. Computations were conducted on the LOEWE-CSC high-performance computer of the State of Hesse.

\section{Author contributions}

A.S. and P.R.S. formulated the initial working hypothesis, D.E. designed the study, D.E., M.S., and J.T. performed all experiments, and M.Š. designed and performed all computations. J.E.P.D. and R.M.K.C. supplied the diamondoids. All authors analyzed the data and co-wrote the manuscript.

\section{Additional information}

Supplementary Information accompanies this paper at https://doi.org/10.1038/s41467018-04843-z.

Competing interests: The authors declare no competing interests.
Reprints and permission information is available online at http://npg.nature.com/ reprintsandpermissions/

Publisher's note: Springer Nature remains neutral with regard to jurisdictional claims in published maps and institutional affiliations.

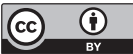

Open Access This article is licensed under a Creative Commons Attribution 4.0 International License, which permits use, sharing, adaptation, distribution and reproduction in any medium or format, as long as you give appropriate credit to the original author(s) and the source, provide a link to the Creative Commons license, and indicate if changes were made. The images or other third party material in this article are included in the article's Creative Commons license, unless indicated otherwise in a credit line to the material. If material is not included in the article's Creative Commons license and your intended use is not permitted by statutory regulation or exceeds the permitted use, you will need to obtain permission directly from the copyright holder. To view a copy of this license, visit http://creativecommons.org/ licenses/by/4.0/.

(c) The Author(s) 2018 